Teply-Elvehjem-Nährmedium, als Sulfonamid wurde Sulfathiazol in Mengen von 12,5-25 $\gamma / \mathrm{ccm}$ verwendet. Leucovorin kam in Mengen von 0,05-0,0005 $\gamma / \mathrm{ccm}$ und Tetrahydro- ${ }^{10} \mathrm{~N}$-formylfolsäure mit 9,01 bis $0,00005 \gamma / \mathrm{ccm}$ zur Anwendung.

\section{Beschreibung der Versuche}

Die mikrobiologischen Teste wurden wie bereits beschrieben durchgeführt 1,6. Die Vorpassage der Bakterien wurde jeweils im optimalen Nährmedium angesetzt und die Versuchsröhrchen daraus mit der Öse beimpft.

2 - A mino-4-oxy-6-pteridylmethyl-

$\left(4^{\prime}-\mathrm{m}\right.$ e thy l- $5^{\prime}-$ oxy a e thy $-\mathrm{thi}$ a zolium) -

bromid

2,8 g 2-Amino-4-oxy-pteridin- $\alpha$-bromessigsäure- $6^{8}$ werden in $10 \mathrm{ccm}$ gesättigter $\mathrm{HBr}$-Eisessig-Lösung $5 \mathrm{Min}$. erhitzt und die Lösung dann unter Feuchtigkeits-Ausschluß im Vakuum eingedampft. Man versetzt jetzt mit $50 \mathrm{ccm}$ wasserfreiem Glykol und $2 \mathrm{~g} 4$-Methyl-5- $\beta$-oxyaethylthiazol und erhitzt unter Rühren und Feuchtigkeitsabschluß 1 Stde. auf $110^{\circ}$. Beim Eingießen in 500

8 R. Ts chesche, F. Korte u. R. Petersen, Chem. Ber. 84, 579 [1951]. ccm Eiswasser fallen 1,5 g des Pteridin-Derivates aus, das durch Umfällen aus $n / 10-\mathrm{NaOH}$ und Kristallisation aus Wasser gereinigt wird.

$$
\begin{aligned}
& \mathrm{C}_{13} \mathrm{H}_{15} \mathrm{~N}_{6} \mathrm{O}_{2} \mathrm{BrS}(396,3) \\
& \text { Ber. S 8,09 Br 25,39. } \\
& \text { Gef. S 7,49 Br 25,10. }
\end{aligned}
$$

Phos phors äure-Ester

$1 \mathrm{~g}$ des vorstehenden Pteridin-Derivates wird mit $20 \mathrm{~g}$ einer im Vakuum bei $150^{\circ}$ entwässerten 85-proz. Phosphorsäure 20 Min. auf $120^{\circ}$ erhitzt. Nach dem Abkühlen wird mit konz. Ammoniaklösung vorsichtig neutralisiert und mit einer Lösung von $\mathrm{AgNO}_{3}$ das Silbersalz gefällt. Man filtriert $a b$ und zerlegt das Salz unter Rühren mit Schwefelwasserstoff. Nach dem Abfiltrieren löst man das Pteridinderivat in $n / 10-\mathrm{NaOH}$ und fällt bei $p_{\mathrm{H}} 4 \mathrm{um}$. Beim Umkristallisieren aus Wasser enthält man $10 \mathrm{mg}$ eines teilweise kristallisierten Produktes, das für die mikrobiologischen Versuche verwendet wurde.

$$
\mathrm{C}_{13} \mathrm{H}_{16} \mathrm{O}_{5} \mathrm{~N}_{6} \mathrm{~S} \text { BrP (476,3) Ber. P 6,50; Gef. 5,80. }
$$

Wir danken der Deutschen Forschungs g e m e in s c h a f $\mathrm{t}$, Bad Godesberg, auch an dieser Stelle für die finanzielle Unterstützung dieser Arbeit.

\title{
Zur Prototropie des Kristallvioletts
}

\author{
Von W. Broser \\ Aus dem Institut für Organische Chemie der Freien Universität Berlin, Berlin-Dahlem \\ (Z. Naturforschg. 10 b, 350-353 [1955]; eingegangen am 28. Februar 1955)
}

\begin{abstract}
Es werden die Prototropie-Gleichgewichte zwischen den einzelnen Ionisationsstufen des Kristallvioletts spektrophotometrisch gemessen. Die mesomeren ein-, zwei- und dreisäurigen Kationen sind miteinander durch Prototropie-Gleichgewichte verbunden, die sich momentan einstellen. Daneben entsteht in einer langsamen Reaktion ein farbloses Carbinolkation, für das eine dreifache positive Ladung ermittelt wurde. Die Gleichgewichtskonstanten für die einzelnen Reaktionen betragen $K_{\mathrm{S}_{1}}=1,09 \cdot 10^{-2} \mathrm{Mol} / l ; K_{\mathrm{S}_{2}}=1,56 \cdot 10^{-1} \mathrm{Mol} / l ; K_{\mathrm{S}_{3}}=$ $1,73 \cdot 10-5(\mathrm{Mol} / l)$ ?
\end{abstract}

In einer früheren Arbeit 1 wurden die Säure-BasenGleichgewichte - die Prototropie-Gleichgewichte - zwischen den einzelnen Ionisationsstufen des Fuchsins untersucht. Dabei wurde gefunden, daß ein sich unmeßbar schnell einstellendes Gleichgewicht zwischen dem 1-säurigen und dem 2-säuriren Kation besteht, und daß in einer langsamen Reaktion ein 3-säuriges Kation gebildet wird. Die einzelnen Ionisationsstufen $\mathrm{F}^{+}, \mathrm{FH}^{++}$und $\mathrm{FH}_{2}{ }^{3}+$ des Fuchsins wurden dabei als mesomere Kationen angenommen (vgl. Tab. 1). Nun erscheint es aber merkwürdig, daß sich das Gleichgewicht zwischen den mesomeren $\mathrm{F}^{+}$und $\mathrm{FH}^{++}$momentan, zwischen $\mathrm{FH}^{++}$und dem ebenfalls als mesomer angesehenen $\mathrm{FH}_{2}{ }^{3}+$ langsam einstellt. Es liegt daher die Vermutung nahe, daß es sich bei dem in langsamer Reaktion gebildeten 3-säurigen

1 W. B r o s e r u. R. H a ve m a n n, Z. Naturforschg. 5 a, 385 [1950].
Ion nicht um das mesomere Kation $\mathrm{FH}_{2} 3+$, sondern um das Carbinolkation $\mathrm{FH}_{3} \cdot \mathrm{OH}^{3}+$ handelt und daß sich infolge ungünstiger spektroskopischer Bedingungen das mesomere 3-säurige Kation $\mathrm{FH}_{2}{ }^{3}+$ der Beobachtung entzogen hat.

Günstiger liegen die experimentellen Bedingungen bei dem analog gebauten Kristallviolett. Hier beobachtet man nämlich beim Übergang zu kleineren $p_{\mathrm{H}}$-Werten ein momentanes Umschlagen der Farbe von Violett über Grün in Gelb, wobei die grüne und gelbe Farbe jedoch nicht beständig sind, sondern allmählich verblaßt. Da das Kristallviolett somit geeignet erscheint, Aufschlüsse über das Verhalten des Fuchsins zu geben, und da andererseits die Prototropie-Verhältnisse des Kristallvioletts genau bekannt sein mußten, ehe wir ${ }^{2}$ die Untersuchung

2 W. B r o s e r u. W. L a u t s c h, Z. Naturforschg. $\mathbf{8}$ b, 711 [1953]. 


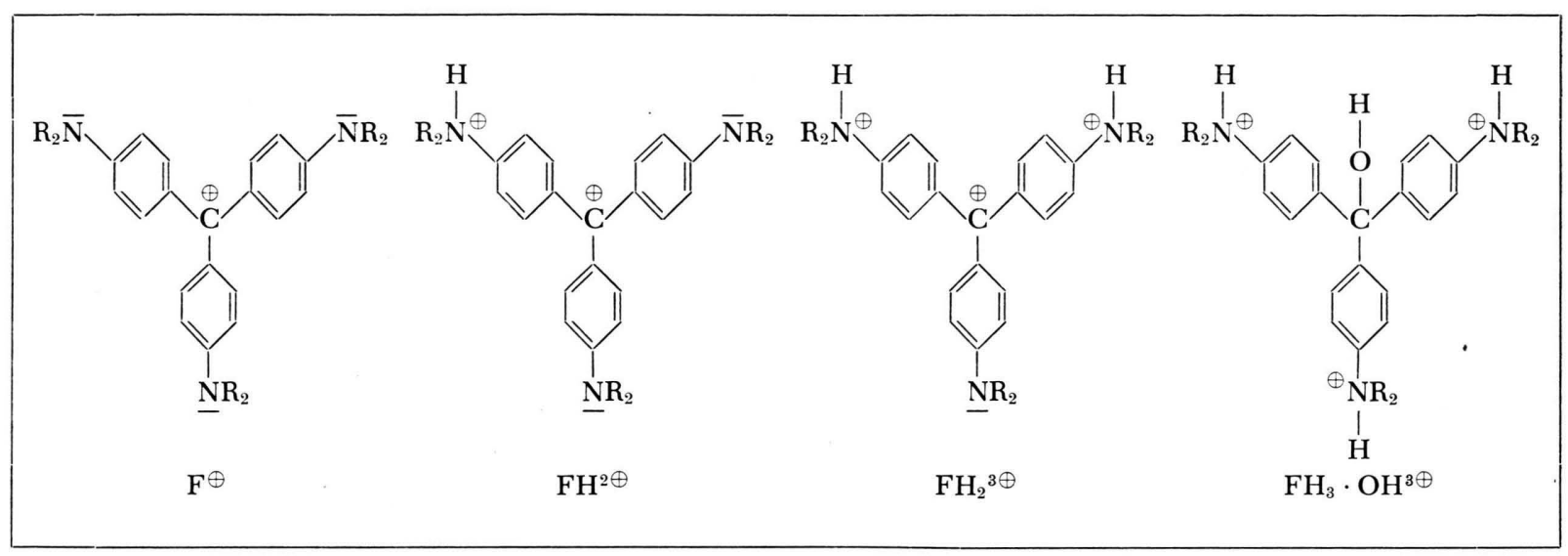

Tab. 1. Übersicht über die verschiedenen Ionisationsstufen. Bei den mesomeren Kationen wurde jeweils nur die Carbeniumgrenzformel angegeben.

seiner Einschlußverbindungen durchführen konnten, studierten wir - wie im Folgenden näher beschrieben die Säure-Base-Gleichgewichte des Kristallvioletts.

Die Untersuchungsmethoden waren die gleichen wie die in der früheren Arbeit ${ }^{1}$ angegebenen, d. h. die Gleichgewichte wurden spektrophotometrisch ermittelt. Die Säuredissoziations-Konstanten $K_{S_{1}}$ und $K_{\mathrm{S}_{2}}$ der Kationensäuren $\mathrm{FH}^{2+}$ bzw. $\mathrm{FH}_{2}{ }^{3+}$ errechnen sich, wie dort abgeleitet wurde, gemäß

$$
\begin{gathered}
\frac{E-E_{\mathrm{FH}}}{E_{\mathrm{F}}-E}=\frac{K_{\mathrm{S}_{1}}}{[\mathrm{H}]}, \\
\frac{E-E_{\mathrm{FH}_{2}}}{E_{\mathrm{FH}}-E}=\frac{K_{\mathrm{S}_{2}}}{[\mathrm{H}]} .
\end{gathered}
$$

$E_{\mathrm{F}}, E_{\mathrm{FH}}$ und $E_{\mathrm{FH}}$ sind dabei die Extinktionen, die gemessen würden, wenn die Gleichgewichte ganz auf der Seite der im Index angegebenen Substanzen liegen würden; $E$ ist die Gleichgewichts-Extinktion.

Da sich im vorliegenden Fall die Gleichgewichte teilweise überschneiden, kann man jedoch nicht diese einfachen Gleichungen zur Ermittlung der K-Werte heranziehen, sondern muß die gegenseitige Beeinflussung berücksichtigen. Tut man dies durch Kombination der aus der Stöchiometrie fließenden Formel mit den einzelnen MWGG., so gelangt man zu der Gleichung:

$$
E=\frac{K_{\mathrm{S}_{1}} \cdot K_{\mathrm{S}_{2}} \cdot E_{\mathrm{F}}+[\mathrm{H}] \cdot K_{\mathrm{S}_{2}} E_{\mathrm{FH}}+[\mathrm{H}]_{2} E_{\mathrm{FH}_{2}}}{K_{\mathrm{S}_{1}} \cdot K_{\mathrm{S}_{2}}+[\mathrm{H}] \cdot K_{\mathrm{S}_{2}}+[\mathrm{H}]^{2}}
$$

Die Extinktionsmessungen werden zweckmäßigerweise bei den Wellenlängen der Absorptionsmaxima der gelben $(423 \mathrm{~m} \mu)$, grünen $(635 \mathrm{~m} \mu)$ und violetten $(592 \mathrm{~m} \mu)$ Ionen durchgeführt; die Kenntnis der Werte von $E_{\mathrm{F}}, E_{\mathrm{FH}}$ und $E_{\mathrm{FH} 2}$ bei je drei Wellenlängen ist also erforderlich. Jedoch sind nur ein Teil davon der direkten Messung zugänglich, $E_{\mathrm{FH}}^{592}, E_{\mathrm{FH}}^{423}, E_{\mathrm{FH}}^{635}$ und $E_{\mathrm{FH}_{2}}^{423}$ müssen als Unbekannte behandelt werden. Mit $K_{\mathbb{S}_{1}}$ und $K_{\mathrm{S}_{2}}$ liegen deshalb insgesamt sechs Unbekannte vor. Gl. (3) muß daher für sechs geeignet gewählte Meßpunkte angesetzt werden.

Eine weitere Schwierigkeit besteht darin, daß die gelben und und grünen Ionen nicht beständig sind. Es wurde daher so gearbeitet, daß eine Stammlösung des Farbstoffs verwendet wurde, die durch ganz geringen Pufferzusatz auf $p_{\mathrm{H}} \sim 5$ gebracht war, bei dem, wie Vorversuche ergaben, aller Farbstoff als einwertiges, violettes Kation vorliegt. Diese Stammlösung wurde schnell durch Vermischen mit einem Puffer bzw. einer entsprechend konz. Salzsäure auf den gewünschten $p_{\mathrm{H}}$-Wert gebracht, dann wurden die Extinktionen bei der gewünschten Wellenlänge in Abhängigkeit von der Zeit gemessen und auf die Zeit Null extrapoliert. Die Farbstoff-Bruttokonzentration betrug in allen Fällen stets $10^{-5} \mathrm{Mol} / l$, die Schichtdicke $2 \mathrm{~cm}$. Die so erhaltenen Werte sind in Tab. 2 angeführt. Die stark sauren Lösungen wurden mittels Salzsäure erzeugt. Die Salzsäurekonzentration in der Meßlösung ist in Spalte 1 angeführt. Spalte 2 gibt die Akivitäts-Koeffizienten und Spalte 3 die damit errechneten Wasserstoffionen-Aktivitäten wieder, aus der nach

$$
p_{\mathrm{H}} \equiv-\log a_{\mathrm{H}}
$$

der $p_{\mathrm{H}}$-Wert ermittelt wurde. Zur Herstellung der weniger sauren Lösungen dienten Glykokoll- und Acetatpuffer, deren $p_{\mathrm{H}}$-Werte mit der Wasserstoff- 


\begin{tabular}{|c|c|c|c|c|c|c|}
\hline$c_{\mathrm{H}}$ & $f_{\mathrm{H}}$ & $a_{\mathrm{H}}$ & $p_{\mathrm{H}}$ & $E_{423}$ & $E_{592}$ & $E_{635}$ \\
\hline 6,4 & 3,75 & 24 & $-1,38$ & 0,500 & - & - \\
\hline 3,2 & 1,40 & 4,5 & $-0,65$ & 0,606 & 0,000 & 0,000 \\
\hline 1,6 & 0,90 & 1,44 & $-0,16$ & 0,620 & - & - \\
\hline 0,8 & 0,79 & 0,63 & $+0,20$ & 0,610 & - & - \\
\hline 0,64 & 0,75 & 0,48 & 0,32 & 0,570 ! & - & 0,194 \\
\hline 0,48 & 0,75 & 0,36 & 0,44 & - & 0,140 & - \\
\hline 0,40 & 0,76 & 0,302 & 0,52 & - & 0,180 & - \\
\hline 0,32 & 0,77 & 0,246 & 0,61 & 0,510 & 0,242 & 0,565 \\
\hline 0,24 & 0,78 & 0,187 & 0,73 & - & 0,380 & \\
\hline 0,16 & 0,78 & 0,125 & 0,90 & 0,424 & 0,474 & 0,972 \\
\hline \multirow[t]{2}{*}{0,08} & 0,81 & 0,665 & 1,19 & 0,350 & 0,735 & 1,285 \\
\hline & & & & 0,320 ! & 0,820 ! & $1,340 !$ \\
\hline \multirow[t]{7}{*}{0,04} & 0,82 & 0,033 & 1,49 & 0,274 & - & 1,360 \\
\hline & & & 1,80 & 0,220 & $1,220 !$ & 1,240 ! \\
\hline & & & 2,50 & 0,090 & 1,700 & 0,745 \\
\hline & & & 3,14 & - & 1,955 & \\
\hline & & & 3,59 & - & 1,960 & \\
\hline & & & 4,40 & - & 1,990 & - \\
\hline & & & 5,11 & 0,000 & 2,030 & 0,344 \\
\hline
\end{tabular}

Tab. 2. Prototropie des Kristallvioletts.

elektrode ermittelt wurden. Durch sechsmaliges Ansetzen von Gl. (3) unter Verwendung der in Tab. 2 mit ! versehenen Werte wurden die unbekannten Extinktionen sowie $K_{S_{1}}$ und $K_{S_{2}}$ berechnet. Es ergaben sich dabei

$$
\begin{aligned}
& K_{\mathrm{S}_{1}}=1,09 \cdot 10^{-2} \mathrm{Mol} / l \text { entspr. } p_{\mathrm{K}_{\mathrm{S}_{1}}}=1,96, \\
& K_{\mathrm{S}_{2}}=1,66 \cdot 10^{-1} \mathrm{Mol} / l \text { entspr. } p_{\mathrm{K}_{\mathrm{S}_{2}}}=0,81 .
\end{aligned}
$$

In Abb. 1 sind die Meßpunkte eingezeichnet; der mit den nunmehr ermittelten unbekannten Extinktionen und Gleichgewichtskonstanten nach Gl. (3) berechnete Kurvenverlauf ist durch die ausgezogenen Linien dargestellt. Die Übereinstimmung mit den Meßpunkten ist gut. Ferner sind die bei der Wellenlänge $592 \mathrm{~m} \mu$ nach drei Tagen, d. h. nach Erreichung des totalen Gleichgewichts, gemessenen Extinktionen eingezeichnet. Die durch sie gelegte gestrichelte Kurve entspricht der Form nach Gl. (2), wobei jedoch [H] im Quadrat steht. Dies bedeutet, daß das Gleichgewicht

$$
\mathrm{FH}_{3} \cdot \mathrm{OH}^{3+} \rightleftharpoons \mathrm{F}^{+}+2 \mathrm{H}^{+}+\mathrm{H}_{2} \mathrm{O}
$$

vorliegt, in der entfärbten sauren Lösung also das 3-säurige Carbinolkation $\mathrm{FH}_{3} \mathrm{OH}^{3+}$ enthalten ist. Aus den Meßpunkten ergibt sich für das obige Gleichgewicht (I)

$$
K_{\mathrm{S}_{3}}=1,73 \cdot 10^{-5}(\mathrm{Mol} / l)^{2} \text { entspr. } p_{\mathrm{KS}_{3}}=4,76 .
$$

Weiter sind in Abb. 1 an der Seite die errechneten Extinktionen, die den einzelnen Ionen zukämen,

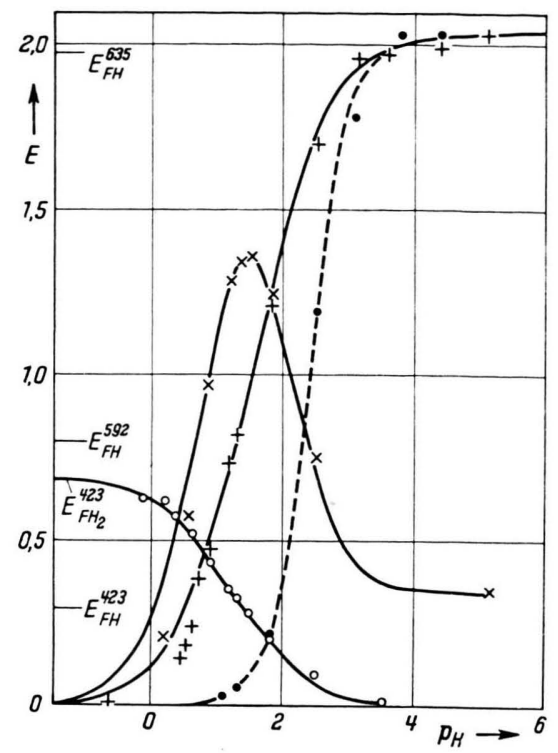

Abb. 1. Die $p_{\mathrm{H}}$-Abhängigkeit der Extinktion. $++\lambda=592 \mathrm{~m} \mu ; t=0$, ○ $\lambda=592 \mathrm{~m} \mu ; t=3 d$. $++\lambda=635 \mathrm{~m} \mu ; t=0, \circ \circ \lambda=423 \mathrm{~m} \mu ; t=0$.

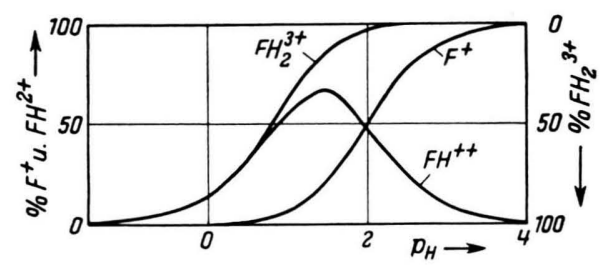

Abb. 2. Die $p_{\mathrm{H}}$-Abhängigkeit der Prozentgehalte an den einzelnen Ionisationsstufen.

wenn sie allein in Lösung vorliegen würden, eingetragen. In Abb. 2 sind die auf Grund der Gleichgewichts-Konstanten berechneten Prozentgehalte der einzelnen Ionen in Abhängigkeit vom $p_{\mathrm{H}}$-Wert aufgetragen. Man erkennt daraus, daß im totalen Gleichgewicht die 2- und 3-säurigen mesomeren Kationen gegenüber dem 3-säurigen Carbinolkation nicht stabil sind, und daß es auch für $t=0$ kein $p_{\mathrm{H}}$-Gebiet gibt, in dem das 2-säurige Kation allein vorliegt.

Aus den Messungen geht weiter hervor, daß die Einstellung der Prototropie-Gleichgewichte zwischen den einzelnen mesomeren Ionisationsstufen beim Kristallviolett unmeßbar rasch erfolgt, wogegen die Einstellung des Gleichgewichts, an dem das Carbinolkation beteiligt ist, langsam verläuft. Eine Übertragung dieser Ergebnisse auf das Fuchsin ergibt, daß es sich bei dem dort ${ }^{1}$ beobachteten 3 -säurigen Kation um das Carbinolkation handelt. 


\section{Reinigung des Kristallvioletts}

Käufliches Kristallviolett wird in viel Wasser gelöst. In die siedende Lösung gibt man so lange in kleinen Portionen 1- $n$. Natronlauge, bis die Carbinolbase ausflockt. Der Niederschlag wird abgesaugt, mit Wasser gewaschen und getrocknet. Dann wird zweimal aus Ligroin umkri- stallisiert. Farblose, sich an der Luft schnell violett färbende Kristalle Schmp. $219^{\circ}$ C. - Diese Carbinolbase diente zur Herstellung der Kristallviolett-Urlösungen, indem sie in Wasser, das durch ganz geringen Pufferzusatz auf $p_{\mathrm{H}} 4,5$ gebracht worden war, im gewünschten Mengenverhältnis gelöst wurde.

\section{NOTIZEN}

\section{Weitere magnetische Untersuchungen zur Struktur der Cyclopentadien- und Inden-Verbindungen der Übergangsmetalle}

Von E. O.Fis cher u. H. Leipfinger

Anorganisch-chemisches und Physikalisches Institut der Technischen Hochschule München

(Z. Naturforschg. 10 b, 353-355 [1955]; eingeg. am 1. April 1955)

Die Annahme, daß es sich bei den Cyclopentadien- und IndenVerbindungen der Übergangsmetalle um Durchdringungskomplexe handelt, konnte durch weitere Untersuchung der magnetischen Eigenschaften erhärtet werden.

Die Bindungsverhältnisse der Cyclopentadien- und Inden-Verbindungen mit Übergangselementen lassen sich durch die Annahme von Druchdringungskomplexen erklären, bei welchen die $3 \pi$-Elektronenpaare der aromatisierten $\left(\mathrm{C}_{5} \mathrm{H}_{5}\right)^{-}$- bzw. $\left(\mathrm{C}_{9} \mathrm{H}_{7}\right)^{-}$-Reste koordinativ in die Schale des zentralen Metallions eintreten ${ }^{1}$.

Bei den magnetischen Untersuchungen wurde die scheinbare Gewichtsänderung der Probe im inhomogenen Magnetfeld mit einer empfindlichen Torsionswaage gemessen und daraus die Molsuszeptibilität der Probesubstanz errechnet. Als Eichsubstanz wurde $\mathrm{CuSO}_{4} \cdot 5 \mathrm{H}_{2} \mathrm{O}$ verwendet. Die Messungen wurden in einem Temperaturbereich von $20^{\circ} \mathrm{K}$ bis $473^{\circ} \mathrm{K}$ durchgeführt. Ergab sich Paramagnetismus, so wurde von den gemessenen Suszeptibilitäten der aus den tabellierten Ionen- bzw. Atominkrementen berechnete diamagnetische Anteil subtrahiert. Aus dem so erhaltenen rein paramagnetischen Anteil wurde mittels des Curie-Weißschen Gesetzes, das mit Ausnahme von $\operatorname{Mn}\left(\mathrm{C}_{5} \mathrm{H}_{5}\right)_{2}$ sehr gut erfüllt wird, das effektive magnetische Moment pro Mol. errechnet.

In den folgenden Tab. 1, 1 a werden die aus der Bindungsvorstellung sich ergebenden effektiven Momente mit den Meßwerten verglichen. In der Spalte (a) sind die vorkommenden Zentralionen aufgeführt, in Spalte (b) deren Elektronenkonfiguration, von der ersten nicht vollbesetzten Schale an. Spalte (c) enthält die Elektronenanordnung des Metalls nach der Komplexbildung und Spalte (d) die somit zu erwartende Zahl ungepaarter Elektronen. Für das magnetische Verhalten der Übergangselemente sind praktisch nur die Spinmomente verantwortlich, da die Bahnmomente durch elektrische Kri-

1 E. O. F is cher u. W. Pf a b, Z. Naturforschg. 7 b, 377 [1952]; E. Ruch u. E. O. Fis cher, Z. Naturforschg. 7 b, 676 [1952]; F. Engelma n n, Z. Naturforschg. 8 b, 775 [1953]. stallfelder völlig zerstört werden. So ergeben sich die in Spalte (e) aufgeführten Momente pro Molekül in Bohrschen Magnetonen

$$
\text { Peff }=\sqrt{4 \mathrm{~S}(\mathrm{~S}+1)},
$$

wenn $S$ die Quantenzahl des Gesamtelektronenspins des Moleküls bedeutet. In Spalte (f) sind die untersuchten Verbindungen angegeben, in Spalte (g) die absoluten Temperaturen, bei denen sie untersucht wurden. Spalte (h) enthält die experimentell bestimmten magnetischen Momente 2 .

Zwischen den erwarteten magnetischen Momenten und den Meßwerten besteht sehr gute Übereinstimmung, mit Ausnahme des $\mathrm{Ni}\left(\mathrm{C}_{9} \mathrm{H}_{7}\right)_{2}$. Doch wurde hier die gleiche Magnetonenzahl auch bei Anwendung der Parawasserstoffmethode gefunden ${ }^{3}$. Wie die Tab. 1 ferner zeigt, wurde für alle Verbindungen, die nach der eingangs kurz erwähnten Bindungsvorstellung Krypton-, Xenon- bzw. Radonkonfiguration für das zentrale Metall ergeben, Diamagnetismus gefunden.

Außer den Verbindungen mit Übergangselementen wurden auch solche mit Magnesium als Zentralion untersucht (Tab. 1 a):

Beide Verbindungen sind als Ionenkomplexe aufzuzufassen, mit Neonkonfiguration des Magnesiumions.

Besonders interessante Verhältnisse liegen bei $\mathrm{Mn}\left(\mathrm{C}_{5} \mathrm{H}_{5}\right)_{2}$ vor ${ }^{4}$. Diese Verbindung wurde bei $20^{\circ} \mathrm{K}, 77^{\circ} \mathrm{K}, 90^{\circ} \mathrm{K}$, $202^{\circ} \mathrm{K}, 292^{\circ} \mathrm{K}, 393^{\circ} \mathrm{K}, 418^{\circ} \mathrm{K}, 433^{\circ} \mathrm{K}, 453^{\circ} \mathrm{K}$ und $473^{\circ} \mathrm{K}$ magnetisch untersucht. Das Ergebnis ist in der Abb. 1 dargestellt. Das $1 / \chi$-T-Diagramm zeigt einen Sprung in den magnetischen Eigenschaften bei $158^{\circ} \mathrm{K}$. Bei dieser Temperatur hat die Substanz einen charakteristischen Umwandlungspunkt; denn die bei tieferen Temperaturen braunen Kristalle nehmen nach dem Überschreiten des Umwandlungspunktes einen hellorange-braunen Farbton an. Dieser Vorgang ist reversibel. Im Gegensatz zu den anderen Cyclopentadien-Verbindungen, die monoklin kristallisieren, haben die braunen $\mathrm{Mn}\left(\mathrm{C}_{5} \mathrm{H}_{5}\right)_{2}$-Kristalle rhombische Struktur.

Es wurden auch isomorphe Mischkristalle von $\mathrm{Mn}\left(\mathrm{C}_{5} \mathrm{H}_{5}\right)_{2}$ (53\%) und $\mathrm{Mg}\left(\mathrm{C}_{5} \mathrm{H}_{5}\right)_{2}$ (47\%) bei den gleichen zehn Temperaturen untersucht. Es ergab sich jedoch, daß hier, nach Abzug der bereits bekannten diamagnetischen Suszepti-

2 Einige der Werte wurden von W. Lechner gemessen.

3 Nach Messungen von J. V o it l ä n d e r, Phys. chem. Institut der Universität München.

${ }_{4}$ Der von F. E n g e lma n n l. c. gemessene Wert jst zu streichen. 